\title{
HYPERTRANSCENDENCY OF PERTURBATIONS OF HYPERTRANSCENDENTAL FUNCTIONS
}

\author{
JIAXING HUANG AND TUEN-WAI NG
}

\begin{abstract}
Inspired by the work of Bank on the hypertranscendence of $\Gamma e^{h}$ where $\Gamma$ is the Euler gamma function and $h$ is an entire function, we investigate when a meromorphic function $f e^{g}$ cannot satisfy any algebraic differential equation over certain field of meromorphic functions, where $f$ and $g$ are meromorphic and entire on the complex plane, respectively. Our results (Theorem 1 and 21) give partial solutions to Bank's Conjecture (1977) on the hypertranscendence of $\Gamma e^{h}$. We also give some sufficient conditions for hypertranscendence of meromorphic function of the form $f+g, f \cdot g$ and $f \circ g$ in Theorem 3 and 4
\end{abstract}

\section{INTRODUCTION AND MAIN RESULTS}

A meromorphic function $f$ on the complex plane is said to be hypertranscendental over a field $\mathcal{K}$ of meromorphic functions, if $f$ does not satisfy any nontrivial algebraic differential equation whose coefficients are in the field $\mathcal{K}$. We are interested in those $\mathcal{K}$ which are related to the growth of $f$. Let $T(r, f)$ be the Nevanlinna characteristic function of $f$ (see Section 2 for the definitions and notations in Nevanlinna theory). We denote by $S(r, f)$ any quantity which is of growth $o(T(r, f))$ as $r \rightarrow \infty$ outside a set of finite measure $E \subset(0, \infty)$. By $\mathcal{M}_{0}$ we mean the field of meromorphic functions $y$ with $T(r, y)=o(r)$ as $r \rightarrow \infty$ outside a set of finite measure and $\mathcal{S}_{f}$ (resp. $\mathcal{S}^{f}$ ) the field of meromorphic functions $y$ satisfying the growth condition $T(r, y)=S(r, f)$ (resp. $T(r, y)=O(T(r, f))$ as $r \rightarrow \infty$ outside a set of finite measure).

In 1887, Hölder [9] established the hypertranscendence of the Euler gamma function $\Gamma$ over the field of rational functions, i.e., $\Gamma$ cannot satisfy any nontrivial algebraic differential equation whose coefficients are rational functions. Hilbert [8], in 1901, proved the hypertranscendence of Riemann zeta function using the functional equation of $\zeta$ and $\Gamma$. In 1976, Bank and Kaufman [4] extended the famous theorems of Hölder and Hilbert by showing that $\Gamma$ and $\zeta$ are hypertranscendental over the field $\mathcal{M}_{0}$. One year later, Bank [2] asked to what extend the hypertranscendence of $\Gamma$ is due to the nature of its poles and zeros. In particular, he posed the following conjecture.

Bank's Conjecture ([2]). For every entire function $h, \Gamma e^{h}$ is hypertranscendental over $\mathcal{M}_{0}$. 
Bank [2] gave an affirmative answer to the above conjecture when either $h$ or $h^{\prime}$ has only finitely many zeros. In 1980, he [3] generalized this result to the following.

Theorem A ([3]). Let $h$ be an entire function with the property that for some nonnegative integer $j$, and some complex number a, the following condition holds :

$$
\bar{N}\left(r, 1 /\left(h^{(j)}-a\right)\right)=S\left(r, h^{(j)}\right),
$$

where as usual, $h^{(0)}$ denotes $h$. Then the function $\Gamma e^{h}$ is hypertranscendental over $\mathcal{M}_{0}$.

Related to Theorem A, we obtained the following.

Theorem 1. Let $h$ be an entire function such that $T\left(r, \Gamma^{\prime} / \Gamma\right)=S\left(r, h^{(j)}\right)$ and

$$
\delta\left(a, h^{(j)}\right)>0
$$

for some $a \in \mathcal{M}_{0}$ and some nonnegative integer $j$. Then $\Gamma e^{h}$ is hypertranscendental over $\mathcal{M}_{0}$.

Related to Bank's Conjecture, we have the following partial result.

Theorem 2. For any entire function $h, P\left(z, \Gamma e^{h} \ldots,\left(\Gamma e^{h}\right)^{(n)}\right) \not \equiv 0$ for any nontrivial distinguished polynomial $P\left(z, u_{0}, \ldots, u_{n}\right)$ over $\mathcal{M}_{0}$.

Remark 1. The notion of distinguished polynomial was first introduced by B. Q. Li and $Z$. Ye in [12]. The definition is given as follow.

Let $I=\left(i_{0}, i_{1}, \ldots, i_{k}\right)$ be a multi-index with $|I|=i_{0}+i_{1}+\cdots+i_{k}$. A polynomial in the variables $u_{0}, u_{1}, \ldots, u_{k}$ with meromorphic function coefficients in a set $S$ can always be written as

$$
P\left(z, u_{0}, u_{1}, \ldots, u_{k}\right)=\sum_{I \in \Lambda} a_{I}(z) u_{0}^{i_{0}} u_{1}^{i_{1}} \cdots u_{k}^{i_{k}}
$$

where the coefficients $a_{I}$ are meromorphic functions in $S$ and $\Lambda$ is an index set. We call $P$ a distinguished polynomial in $u_{0}, u_{1}, \ldots, u_{k}$ with coefficients in $S$, or simply an $S$-distinguished polynomial, if the index set $\Lambda$ satisfies $\left|I_{i}\right| \neq\left|I_{j}\right|$ for any distinct indices $I_{i}, I_{j}$ in $\Lambda$. In other words, each homogeneous part of the distinguished polynomial $P$ contains one term only.

If $\mathcal{K}$ is a field of meromorphic functions, we denote by $A(\mathcal{K})$ the set of all meromorphic functions which satisfy some algebraic differential equation over $\mathcal{K}$. It is well known (see Chapter 14 of [10]) that $A(\mathcal{K})$ is a differential field, i.e., a field with an additional map $D: A(\mathcal{K}) \rightarrow A(\mathcal{K})$ such that $D(a \cdot b)=(D a) \cdot b+a \cdot D b$ for any $a, b \in A(\mathcal{K})$.

To explain the difference between Theorem A and Theorem 1, let us sketch the main idea of the proof of Theorem A (see Part B in [3] or Chapter 14 of [10]). 
Let $h$ be an entire function satisfying the assumption (1) in Theorem A. If $\Gamma e^{h} \in$ $A\left(\mathcal{M}_{0}\right)$ and $a \in \mathbb{C}$, set $g=h-\left(a z^{j} / j !\right)$ which satisfies the condition $\bar{N}\left(r, 1 / g^{(j)}\right)=$ $S\left(r, g^{(j)}\right)$. Applying Lemma A below and using the fact that $T\left(r, \Gamma^{\prime} / \Gamma\right)=r+o(r)$, one can conclude that $T\left(r, g^{(j)}\right)=O(r)$. On the other hand, $g$ is an entire function with $\bar{N}\left(r, 1 / g^{(j)}\right)=S\left(r, g^{(j)}\right)$, thus $T\left(r, g^{(j+1)} / g^{(j)}\right)=o(r)$. Hence $g^{(j+1)} / g^{(j)}$ belongs to $\mathcal{M}_{0}$ which implies $g \in A\left(\mathcal{M}_{0}\right)$. Thus $h$ and $h^{\prime} \in A\left(\mathcal{M}_{0}\right)$. Since $h^{\prime}=\left(e^{h}\right)^{\prime} /\left(e^{h}\right)$, it follows that $e^{h} \in A\left(\mathcal{M}_{0}\right)$. Combining with the assumption that $\Gamma e^{h} \in A\left(\mathcal{M}_{0}\right)$, one can deduce a contradiction to the hypertranscendence of $\Gamma$ over $\mathcal{M}_{0}$.

Actually, from the proof of Theorem A, it is not hard to see that the assumption $\Gamma e^{h} \in A\left(\mathcal{M}_{0}\right)$ and the condition (1) imply that $T\left(r, h^{(j)}\right)=O\left(T\left(r, \Gamma^{\prime} / \Gamma\right)\right)$. Our Theorem 1 considers a sort of complement assumption that $T\left(r, \Gamma^{\prime} / \Gamma\right)=S\left(r, h^{(j)}\right)$. Under this assumption, the condition (2) is less restrictive than the one on $\bar{N}\left(r, 1 /\left(h^{(j)}-\right.\right.$ $a)$ ) in Theorem A. In addition, $a$ can also be nonconstant.

To produce more examples of hypertranscendental functions, Bank also investigated the hypertranscendency of the perturbation of hypertranscendental meromorphic functions by adding a small function.

Theorem B ([3]). Let $f$ be a meromorphic function on the complex plane which is hypertranscendental over a differential field $\mathcal{S} \subset \mathcal{S}_{f}$. Let $g$ be a meromorphic function on the complex plane. Then, if $f+g$ satisfies an algebraic differential equation over $\mathcal{S}$, we have

$$
T(r, f)=O(\bar{N}(r, 1 / f)+\bar{N}(r, f)+T(r, g))
$$

as $r \rightarrow \infty$ outside of a possible exceptional set of finite measure.

In particular, if all $\bar{N}(r, 1 / f), \bar{N}(r, f)$ and $T(r, g)$ are $S(r, f)$, then $f+g$ must be hypertranscendental over $\mathcal{S}$.

The proofs of Theorem A and B in [2, 3] depend on the following Lemma first appeared in [1].

Lemma A ([1]). Let $P\left(z, y, y^{\prime}, \ldots, y^{(n)}\right)$ be a polynomial in $y, y^{\prime}, \ldots, y^{(n)}$ whose coefficients are meromorphic functions on $\mathbb{C}$. For each $r>0$, let $\Delta(r)$ be the maximum of the Nevanlinna characteristics of the coefficients of $P$. Let $f$ be a nonzero meromorphic function on the complex plane satisfying the equation $P=0$, but for some nonnegative integer $q, P_{q}\left(f, f^{\prime}, \ldots, f^{(n)}\right) \neq 0$, where $P_{q}$ is the homogeneous part of $P$ of total degree $q$ in the indeterminates $y, y^{\prime}, \ldots, y^{(n)}$. Then

$$
T(r, f)=O(E(r))
$$

as $r \rightarrow \infty$, outside of a possible exceptional set of finite measure, where

$$
E(r)=\bar{N}(r, 1 / f)+\bar{N}(r, f)+\Delta(r)+\log r .
$$

In addition, for any $\alpha>1$, there exist positive constants $c$ and $r_{0}$ such that

$$
T(r, f) \leq c E(\alpha r), \text { for all } r \geq r_{0} .
$$


In 1991, Y. Z. He and C. C. Yang [7] proved that $\Gamma(g)$ is hypertranscendental over the field $\mathcal{M}^{g}$ of meromorphic functions $y$ with $T(r, y)=O(T(r, g))$ by using Steinmetz's Reduction Theorem (Theorem C below). Their method can be applied to the general case (see Theorem [3). In 2007, Markus [13] applied the method of differential algebra to obtain the hypertranscendence of $\zeta(\sin z)$ and $\Gamma(\sin z)$ over $\mathbb{C}$, and he proved the differential independence between $f_{i}$ and $f_{j}(\sin z)$ for $i, j=1,2$, where $f_{1}=\Gamma$ and $f_{2}=\zeta$.

Applying the same idea of He and Yang in [7, we obtain the following general result which covers the results of He and Yang [7].

Theorem 3. Let $f$ be hypertranscendental over the rational function field $\mathbb{C}(z)$ and $g$ be a nonconstant entire function. Then $f \circ g$ is hypertranscendental over the field $\mathcal{S}^{g}$.

As a consequence, we can generalize a result of L. Markus (see Lemma 1 in [13]) by using a different method.

Corollary 1. Let a be a nonzero complex number. Then both $\Gamma(\sin a z)$ and $\zeta(\sin a z)$ are hypertranscendental over the field of meromorphic functions y with $T(r, y)=$ $O(r)$ as $r \rightarrow \infty$ outside some set of finite measure.

It is natural to consider the hypertranscendency of $g \circ f$ over some fields for entire hypertranscendental $f$ and meromorphic $g$. This seems to be a more difficult problem as Steinmetz's Reduction Theorem cannot be applied directly here (see Remark 2 in Section 3). However, we do obtain one related result in Theorem 4(1).

Inspired by the results of Bank, He-Yang and Markus, in this paper, we will first prove a result similar to Lemma A, that is $T(r, f)$ can be controlled by one counting function $N(r, 1 / f)$ (see Lemma 2). Using Lemma 2, we then obtain the following results on the hypertranscendency of perturbations of hypertranscendental functions, including that of $\Gamma$ and $\Gamma e^{h}$.

Theorem 4. Let $g$ and $f$ be meromorphic functions and $\mathcal{S}$ be the field of meromorphic functions y with $T(r, y)=S\left(r, f^{\prime} / f\right)$, i.e. $\mathcal{S}=\mathcal{S}_{f^{\prime} / f}$. Let $\mathcal{O}$ be the set of entire functions on $\mathbb{C}$. Suppose $f$ is hypertranscendental over $\mathcal{S}$.

(1) If $f \in \mathcal{O}$, and $g-R$ has finitely many zeros, where $R$ is a non-constant rational function, then $g \circ f$ is hypertranscendental over $\mathcal{S}$.

(2) Assume that $f \in \mathcal{S}_{g}$ and $\delta(a, g)>0$ for some $a \in \mathcal{S} \backslash\{0\}$, then $f g$ is hypertranscendental over $\mathcal{S}$.

(3) If there exists a non-negative integer $k$ such that $T(r, f)=S\left(r, g^{(k)}\right)$ and $\delta\left(a, g^{(k)}\right)>$ 0 for some $a \in \mathcal{S}$, then $f+g$ is hypertranscendental over $\mathcal{S}$.

(4) Assume that $g \in \mathcal{O}$, and if there exists a nonnegative integer $k$ such that $T\left(r, f^{\prime} / f\right)=S\left(r, g^{(k)}\right)$ and

$$
\delta\left(a, g^{(k)}\right)>0
$$


HYPERTRANSCENDENCY OF PERTURBATIONS OF HYPERTRANSCENDENTAL FUNCTIONS

for some $a \in \mathcal{S}$, then $f e^{g}$ is hypertranscendental over $\mathcal{S}$.

(5) If $g \in \mathcal{O}$ and $f \in \mathcal{S}_{\exp (g)}$, then $P\left(z, f e^{g},\left(f e^{g}\right)^{\prime}, \ldots,\left(f e^{g}\right)^{(n)}\right) \not \equiv 0$ for any nontrivial distinguished polynomial $P\left(z, u_{0}, \ldots, u_{n}\right)$ over $\mathcal{S}$.

In Section 5, we will use Theorem4to prove Theorem11and2, Section 2 introduces the basics of Nevanlinna Theory. Theorem 3 and 4 will be proven in Section 3 and 4, respectively.

\section{Nevanlinna TheOrY}

We recall the basic notations and results of Nevanlinna theory [10] which are main tools for proving our results.

Let $f$ and $a$ be meromorphic functions in the complex plane $\mathbb{C}$ and $\mathbb{D}_{r}=\{|z|<r\}$. Denote the number of poles of $f$ in $\mathbb{D}_{r}$ by $n(r, f)$, and let $n(r, a)=n(r, a, f)=$ $n(r, 1 /(f-a))$. When the number of distinct poles of $f$ in $\mathbb{D}_{r}$ is denoted by $\bar{n}(r, f)$, we then let $\bar{n}(r, a)=\bar{n}(r, 1 /(f-a))$. Correspondingly we define the counting function and truncated counting function in Nevanlinna theory as follows:

$$
\begin{aligned}
& N(r, a, f):=\int_{0}^{r} \frac{n(t, a)-n(0, a)}{t} d t+n(0, a) \log r \\
& \bar{N}(r, a, f):=\int_{0}^{r} \frac{\bar{n}(t, a)-\bar{n}(0, a)}{t} d t+\bar{n}(0, a) \log r .
\end{aligned}
$$

The proximity function is defined as

$$
m(r, f):=\frac{1}{2 \pi} \int_{0}^{2 \pi} \log ^{+}\left|f\left(r e^{i \theta}\right)\right| d \theta
$$

and

$$
m(r, a, f):=m(r, 1 /(f-a)),
$$

where $\log ^{+} x=\max \{0, \log x\}$. The Nevanlinna characteristic function of $f$ is defined by

$$
T(r, f)=m(r, f)+N(r, f) .
$$

The First Main Theorem of Nevanlinna theory for small functions [14] says that for any meromorphic function $a$ with $T(r, a)=S(r, f)$,

$$
T(r, f)=T(r, a, f)+S(r, f)
$$

where $T(r, a, f):=m(r, a, f)+N(r, a, f)$. Finally, we denote the Nevanlinna order of $f$ by

$$
\rho(f):=\limsup _{r \rightarrow \infty} \frac{\log T(r, f)}{\log r}
$$

and the deficiency of $a$ for $f$ by

$$
\delta(a, f):=\liminf _{r \rightarrow \infty} \frac{m(r, a, f)}{T(r, f)}=1-\limsup _{r \rightarrow \infty} \frac{N(r, a, f)}{T(r, f)} .
$$


If $\delta(a, f)>0$, then we say $a$ is a deficient function of $f$.

The logarithmic derivative lemma states that

Lemma 1 ([10]). Let $f$ be a transcendental meromorphic function and $k \geq 1$ be an integer. Then

$$
m\left(r, \frac{f^{(k)}}{f}\right)=S(r, f),
$$

and if $f$ is of finite order of growth, then

$$
m\left(r, \frac{f^{(k)}}{f}\right)=O(\log r) .
$$

\section{Proof of Theorem 3}

To prove Theorem [3, we first introduce the Steinmetz's Reduction Theorem.

Theorem C (Steinmetz's Reduction Theorem [6, 15]). Let $F_{j}, 1 \leq j \leq N$ be meromorphic functions on $\mathbb{C}$. Let $h_{j}, 1 \leq j \leq N$ be meromorphic and $g$ be entire on $\mathbb{C}$ such that for each $j$,

$$
T\left(r, h_{j}\right)=O(T(r, g))
$$

as $r \rightarrow \infty$ outside some set of finite measures. Given a functional equation of the form

$$
F_{1}(g(z)) h_{1}(z)+\cdots+F_{N}(g(z)) h_{N}(z)=0,
$$

then there exist polynomials $p_{j}$, not all zeros, such that

$$
p_{1}(g(z)) h_{1}(z)+\cdots+p_{N}(g(z)) h_{N}(z)=0 .
$$

Furthermore, if $h_{j} \not \equiv 0$ for some $j$, then there exist polynomials $Q_{j}$, not all zeros, such that

$$
F_{1}(z) Q_{1}(z)+\cdots+F_{N}(z) Q_{N}(z)=0 .
$$

Proof of Theorem 3. We will follow the idea of the proof of Theorem 4 in [7].

Suppose that $f \circ g$ satisfies a nontrivial algebraic differential equation with coefficients in $\mathcal{S}^{g}$, i.e., there exists a nontrivial differential polynomial $P\left(z, w, w^{\prime}, \ldots, w^{(n)}\right)$ with coefficients in $\mathcal{S}^{g}$ such that

$$
P\left(z, f \circ g,(f \circ g)^{\prime}, \ldots,(f \circ g)^{(n)}\right)=\sum_{j}\left(M_{j}(f) \circ g\right)\left(H_{j}(g)(z)\right)=0
$$

where $M_{j}(f)$ is a differential monomial of $f$ with constant coefficients and $H_{j}(g)(z)$ is a differential polynomial of $g(z)$ whose coefficients are some linear combinations of the coefficients of the original differential polynomial $P\left(z, w, w^{\prime}, \ldots, w^{(n)}\right)$.

Now, set $F_{j}(z)=M_{j}(f)(z)$ and $h_{j}(z)=H_{j}(g)(z)$, it follows from the second result of Theorem $\mathrm{C}$ that there exist polynomials $Q_{i}$, not all zeros, such that

$$
F_{1}(z) Q_{1}(z)+\cdots+F_{N}(z) Q_{N}(z)=0 .
$$


which implies that $f$ satisfies a nontrivial algebraic differential equation with coefficients in $\mathbb{C}(z)$. This is a contradiction to our assumption that $f$ is hypertranscendental over $\mathbb{C}(z)$.

Remark 2. Here, we will explain the reason why the Steinmetz's Reduction Theorem does not work for the hypertranscendency of $g \circ f$. We use the same idea of proof of Theorem 3. Suppose that $g \circ f$ satisfies a nontrivial algebraic differential equation over a suitable field such that we can apply the Steinmetz's Reduction Theorem, thus we have

$$
p_{1}(f(z)) h_{1}(z)+\cdots+p_{N}(f(z)) h_{N}(z)=0
$$

or

$$
F_{1}(z) Q_{1}(z)+\cdots+F_{N}(z) Q_{N}(z)=0
$$

where $h_{j}(z)$ is a differential polynomial of $f(z)$ whose coefficients are some linear combinations of the coefficients of the algebraic differential equation $g \circ f$ satisfied, and $F_{j}(z)$ is a differential monomial of $g$ with constant coefficients. From these two equalities, we cannot deduce any contradictions even through we have known the hypertranscendency of $f$.

\section{Proof of Theorem 4}

In this section, we are now going to prove our main result (Theorem 4). To prove Theorem 4, we need the following lemmata.

Lemma 2. Let $f$ be a nonzero meromorphic function on the complex plane and $P\left(z, y, y^{\prime}, \ldots, y^{(n)}\right)$ be a polynomial in $y, y^{\prime}, \ldots, y^{(n)}$ whose coefficients are in the field $\mathcal{S}_{f}$. Suppose $f$ satisfies the equation $P=0$. Rewrite $P=0$ as $P_{q}=\sum_{j=k}^{m} P_{j}$, for some nonnegative integers $q$ and $k(>q)$ such that $P_{q} \neq 0$ for each $j \geq k$, where $P_{j}$ is the homogeneous part of $P$ of total degree $j$ in the indeterminates $y, y^{\prime}, \ldots, y^{(n)}$. Then for any integer $N$ with $q \leq N \leq k$,

$$
m\left(r, P_{q} / f^{N}\right)=S(r, f) .
$$

In addition if $q=0$, then

$$
T(r, f)=N(r, 0, f)+S(r, f) .
$$

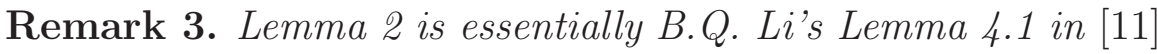

Proof of Lemma Q Let $P\left(z, u_{0}, \ldots, u_{n}\right)$ be a polynomial in $u_{0}, \ldots, u_{n}$ with coefficients in $\mathcal{S}_{f}$. Assume that

$$
I=\left\{i:=\left(i_{0}, i_{1}, \ldots, i_{n}\right) \mid i_{j} \text { is a nonnegative integer and } 0 \leq j \leq n\right\}
$$

is an index set with finite cardinal numbers. Define

$$
|i|=\sum_{j=0}^{n} i_{j} \quad \text { and } \quad I_{p}=\{i \in I:|i|=p\} .
$$


For each $l \geq q$, let

$$
P_{l}=\sum_{i \in I_{l}} a_{i}(z) u_{0}^{i_{0}} \ldots u_{n}^{i_{n}}
$$

where $a_{i} \in \mathcal{S}_{f}$.

Take any point $z \in \mathbb{C}$, we consider several cases.

Case (i) $|f(z)| \geq 1$. Since $P_{q}=\sum_{i \in I_{q}} a_{i}(z) u_{0}^{i_{0}} \ldots u_{n}^{i_{n}}$,

$$
\begin{aligned}
\left|\frac{P_{q}\left(f, f^{\prime}, \ldots, f^{(n)}\right)}{f^{N}}(z)\right| & \leq\left|\frac{P_{q}\left(f, f^{\prime} \ldots, f^{(n)}\right)}{f^{q}}(z)\right| \\
& \leq \sum_{i \in I_{q}}\left|a_{i}(z) \frac{f^{i_{0}}\left(f^{\prime}\right)^{i_{1}} \cdots\left(f^{(n)}\right)^{i_{m}}}{f^{q}}\right|:=G_{1}(z) .
\end{aligned}
$$

Case (ii) $|f(z)| \leq 1$. Then by $P_{q}=\sum_{j=k}^{m} P_{j}, q \leq N \leq k$, we have

$$
\begin{aligned}
\left|\frac{P_{q}\left(f, f^{\prime}, \ldots, f^{(n)}\right)}{f^{N}}(z)\right| & =\left|\sum_{j=k}^{m} \frac{P_{j}\left(f, f^{\prime} \ldots, f^{(n)}\right)}{f^{j}}(z) f^{j-N}\right| \\
& \leq \sum_{j=k}^{m}\left|\frac{P_{j}\left(f, f^{\prime} \ldots, f^{(n)}\right)}{f^{j}}(z)\right||f|^{j-N} \\
& \leq \sum_{j=k}^{m} \sum_{i \in I_{j}}\left|a_{i}(z) \frac{f^{i_{0}}\left(f^{\prime}\right)^{i_{1}} \cdots\left(f^{(n)}\right)^{i_{m}}}{f^{j}}\right|:=G_{2}(z) .
\end{aligned}
$$

Combining the above results, we see that in any case

$$
\left|\frac{P_{q}\left(f, f^{\prime}, \ldots, f^{(n)}\right)}{f^{N}}(z)\right| \leq G_{1}(z)+G_{2}(z)
$$

for any $z \in \mathbb{C}$. By the well-known Logarithmic Derivative Lemma and $a_{i} \in \mathcal{S}_{f}$, we deduce that

$$
m\left(r, P_{q} / f^{N}\right) \leq m\left(r, G_{1}+G_{2}\right)=S(r, f) .
$$

Now if $q=0$, then by taking $N=1$, we have

$$
m(r, 1 / f) \leq m\left(r, P_{0} / f\right)+m\left(r, 1 / P_{0}\right)+O(1)=S(r, f)
$$

as $T\left(r, P_{0}\right)=S(r, f)$. Hence the result follows from the First Main Theorem of Nevanlinna theory.

As a consequence, one can also obtain the following lemma first proved by A. Mohon'ko in 1982.

Lemma 3 ([14]). Let $f$ be a transcendental meromorphic solution of an algebraic differential equation $P(y)=P\left(z, y, y^{\prime}, \ldots, y^{(k)}\right)=0$ with coefficients in $\mathcal{S}_{f}$. If a 
meromorphic function $\phi$ with $T(r, \phi)=S(r, f)$ does not solve $P\left(z, y, y^{\prime}, \ldots, y^{(k)}\right)=0$ i.e. $P\left(z, \phi, \phi^{\prime}, \ldots, \phi^{(k)}\right) \not \equiv 0$, then

$$
m\left(r, \frac{1}{f-\phi}\right)=S(r, f)
$$

Proof. Let $g=f-\phi$, then $T(r, g)=T(r, f)+S(r, f)$. Since $P(f) \equiv 0$, we have

$$
P(f)=P(g+\phi)=Q(g)+P(\phi) \equiv 0
$$

where $Q$ is a differential polynomial over $\mathcal{S}_{f}$ with lowest degree at least one, as $T(r, \phi)=S(r, f)$. The result follows immediately from Lemma 2 as $P(\phi) \not \equiv 0$.

Lemma 4 ([5]). Let $f$ be a transcendental entire function and let $g$ be a transcendental meromorphic function in the complex plane, then $T(r, f)=o(T(r, g \circ f))$ as $r \rightarrow \infty$.

Proof of Theorem 4. 1). Without loss of generality, we can assume $R(z)=z$, since if $f$ is hypertranscendental over $\mathcal{S}$, it is easy to show that $R \circ f$ is also hypertranscendental over $\mathcal{S}$.

Suppose $g(z)-z=0$ has $d$ roots, then $g(z)-z=Q(z) A(z)$ where $Q$ is a polynomial with degree $d$, and $A$ is a transcendental meromorphic function which is nowhere zero. Hence if $f$ is an entire function, we have

$$
N(r, 0, g \circ f-f)=N(r, 0, Q(f) A(f))=N(r, 0, Q(f)) \leq d T(r, f)+S(r, f) .
$$

By Lemma 4, we have $T(r, f)=o(T(r, g \circ f))$. Suppose $g \circ f$ is not hypertranscendental over $\mathcal{S}$, that is, $g \circ f$ is a solution of an algebraic differential equation $P\left(z, y, y^{\prime}, \ldots, y^{(k)}\right)=0$ with coefficients in $\mathcal{S}$ (hence in $\mathcal{S}_{g \circ f}$ as well). By Lemma 3 and the assumption that $f$ is hypertranscendental over $\mathcal{S}$, we have

$$
m\left(r, \frac{1}{g \circ f-f}\right)=S(r, g \circ f) .
$$

By the First Main Theorem of Nevanlinna Theory for small functions [14,

$$
\begin{aligned}
T(r, g \circ f) & =T(r, g \circ f-f)+S(r, g \circ f) \\
& =m(r, 0, g \circ f-f)+N(r, 0, g \circ f-f)+S(r, g \circ f) \\
& \leq S(r, g \circ f)+d T(r, f)=S(r, g \circ f)
\end{aligned}
$$

which is a contradiction. This completes the proof of the first part.

2). If $a \not \equiv 0$, since $f$ is hypertranscendental over $\mathcal{S}$, it is easy to show that $a f$ is also hypertranscendental over $\mathcal{S}$, as $a \in \mathcal{S}$.

Since $T(r, f)=S(r, g), T(r, a)=S\left(r, f^{\prime} / f\right)=S(r, f)$, one can obtain that $T(r, a f)=T(r, f)+S(r, f)=S(r, f g)$.

Suppose $f g$ is not hypertranscendental over $\mathcal{S}$, that is, $f g$ is a solution of an algebraic differential equation $P\left(z, y, y^{\prime}, \ldots, y^{(k)}\right)=0$ with coefficients in $\mathcal{S}$ (hence 
in $\mathcal{S}_{f g}$ also). By Lemma 3 and the hypertranscendence of af over $\mathcal{S}$, we have

$$
m\left(r, \frac{1}{f g-a f}\right)=S(r, f g)=S(r, g) .
$$

On the other hand, by the First Main Theorem of Nevanlinna Theory for small functions, as $T(r, a f)=S(r, f g)$,

$$
\begin{aligned}
T(r, f g) & =T(r, f g-a f)+S(r, f g) \\
& =m(r, 0, f g-a f)+N(r, 0, f g-a f)+S(r, f g) \\
& \leq N(r, 0, g-a)+N(r, 0, f)+S(r, g) \\
& =N(r, 0, g-a)+S(r, g) .
\end{aligned}
$$

Since $T(r, f g)=T(r, g)+S(r, g)$, it follows that $T(r, g)=N(r, a, g)+S(r, g)$ which is a contradiction to the assumption that $\delta(a, g)>0$.

3). If $f+g \in A(\mathcal{S})$, so does $f^{(k)}+g^{(k)}$, that is, there exists a nontrivial algebraic differential equation $P\left(z, y, y^{\prime}, \ldots, y^{(n)}\right)=0$ over $\mathcal{S}$ such that

$$
P\left(z, f^{(k)}+g^{(k)}, f^{(k+1)}+g^{(k+1)}, \ldots, f^{(k+n)}+g^{(k+n)}\right) \equiv 0 .
$$

Set

$$
Q\left(z, g^{(k)}, g^{(k+1)}, \ldots, g^{(n+k)}\right):=P\left(z, f^{(k)}+g^{(k)}, f^{(k+1)}+g^{(k+1)}, \ldots, f^{(k+n)}+g^{(k+n)}\right),
$$

then $Q\left(z, g^{(k)}, g^{(k+1)}, \ldots, g^{(n+k)}\right) \equiv 0$. It is easy to check that all the Nevanlinna characteristic functions of the coefficients of $Q\left(z, g^{(k)}, g^{(k+1)}, \ldots, g^{(n+k)}\right)$ are $S\left(r, g^{(k)}\right)$, as $T(r, f)=S\left(r, g^{(k)}\right)$ and $T\left(r, f^{(k)}\right) \leq(k+1) T(r, f)+S(r, f)$

On the other hand, since $f$ is hypertranscendental over $\mathcal{S}$, so is $f^{(k)}+a$ for any $a \in \mathcal{S}$, hence

$$
Q\left(z, a, a^{\prime}, \ldots, a^{(n)}\right)=P\left(z, f^{(k)}+a, f^{(k+1)}+a^{\prime}, \ldots, f^{(k+n)}+a^{(n)}\right) \not \equiv 0 .
$$

By Lemma 3, we have

$$
m\left(r, \frac{1}{g^{(k)}-a}\right)=S\left(r, g^{(k)}\right)
$$

which is a contradiction to the assumption that $\delta\left(a, g^{(k)}\right)>0$ for some $a \in \mathcal{S}$.

4). If $f e^{g} \in A(\mathcal{S})$, then clearly, $\frac{f^{\prime}}{f}+g^{\prime}=\frac{\left(f e^{g}\right)^{\prime}}{f e^{g}} \in A(\mathcal{S})$, and hence so does $\left(\frac{f^{\prime}}{f}\right)^{(k)}+g^{(k+1)}$ for any nonnegative integer $k$, that is, there exists an algebraic differential equation $P\left(z, y, y^{\prime}, \ldots, y^{(n)}\right)=0$ over $\mathcal{S}$ such that

$$
P\left(z,\left(\frac{f^{\prime}}{f}\right)^{(k)}+g^{(k+1)},\left(\frac{f^{\prime}}{f}\right)^{(k+1)}+g^{(k+2)}, \ldots,\left(\frac{f^{\prime}}{f}\right)^{(k+n)}+g^{(k+n+1)}\right) \equiv 0 .
$$


Set

$Q\left(z, g^{(k)}, g^{(k+1)}, \ldots, g^{(n+k+1)}\right):=P\left(z,\left(\frac{f^{\prime}}{f}\right)^{(k)}+g^{(k+1)}, \ldots,\left(\frac{f^{\prime}}{f}\right)^{(k+n)}+g^{(k+n+1)}\right)$,

then

$$
Q\left(z, g^{(k)}, g^{(k+1)}, \ldots, g^{(n+k+1)}\right) \equiv 0
$$

and all the Nevanlinna characteristic functions of the coefficients of $Q\left(z, g^{(k)}, g^{(k+1)}, \ldots\right.$, $\left.g^{(n+k+1)}\right)$ are $S\left(r, g^{(k)}\right)$ from

$$
T\left(r, f^{\prime} / f\right)=S\left(r, g^{(k)}\right)
$$

and

$$
T\left(r,\left(f^{\prime} / f\right)^{(j)}\right) \leq(j+1) T\left(r, f^{\prime} / f\right)+S\left(r, f^{\prime} / f\right)
$$

for any nonnegative integer $j$.

On the other hand, since $f$ is hypertranscendental over $\mathcal{S}$, so is $\left(f^{\prime} / f\right)^{(k)}$ for any nonnegative integer $k$, and hence so is $\left(f^{\prime} / f\right)^{(k)}+a^{\prime}$ for any $a \in \mathcal{S}$. Therefore,

$Q\left(z, a, a^{\prime}, \ldots, a^{(n+1)}\right)=P\left(z,\left(\frac{f^{\prime}}{f}\right)^{(k)}+a^{\prime},\left(\frac{f^{\prime}}{f}\right)^{(k+1)}+a^{\prime \prime}, \ldots,\left(\frac{f^{\prime}}{f}\right)^{(k+n)}+a^{(n+1)}\right) \not \equiv 0$.

By Lemma 3, we have

$$
m\left(r, \frac{1}{g^{(k)}-a}\right)=S\left(r, g^{(k)}\right)
$$

which is a contradiction to the inequality (3).

5). Let

$$
P\left(z, u_{0}, u_{1}, \ldots, u_{n}\right)=\sum_{i=0}^{m} P_{i}\left(z, u_{0}, u_{1}, \ldots, u_{n}\right)
$$

be a distinguished polynomial over $\mathcal{S}$, where $P_{i}\left(z, u_{0}, u_{1}, \ldots, u_{n}\right)$ contains only one term $a_{i}(z) u_{0}^{i_{0}} u_{1}^{i_{1}} \cdots u_{n}^{i_{n}}$ with coefficient $a_{i} \in \mathcal{S}$ and $i=i_{0}+i_{1}+\cdots+i_{n}$.

We first notice that the assumption $f \in \mathcal{S}_{\exp (g)}$ and Lemma 4 imply that

$$
\begin{aligned}
T\left(r, \frac{\left(f e^{g}\right)^{\prime}}{f e^{g}}\right) & =T\left(r, \frac{f^{\prime}}{f}+g^{\prime}\right) \\
& \leq 2 T(r, f)+S(r, f)+2 T(r, g)+S(r, g)=S\left(r, f e^{g}\right)
\end{aligned}
$$

Assume to the contrary that $P\left(z, f e^{g},\left(f e^{g}\right)^{\prime}, \ldots,\left(f e^{g}\right)^{(n)}\right) \equiv 0$. Let $q$ be a nonnegative integer such that $a_{q} \neq 0$ and $a_{j} \equiv 0, j=0,1, \ldots, q-1$. Applying Lemma 2 to $N=q+1$, one can conclude that

$$
m\left(r, P_{q} /\left(f e^{g}\right)^{q+1}\right)=S\left(r, f e^{g}\right)
$$

hence $m\left(r, 1 /\left(f e^{g}\right)\right)=S\left(r, f e^{g}\right)$ as $T\left(r, P_{q} /\left(f e^{g}\right)^{q}\right)=S\left(r, f e^{g}\right)$. However, $m\left(r, 0, f e^{g}\right)+$ $N\left(r, 0, f e^{g}\right)=S\left(r, f e^{g}\right)+N(r, 0, f) \leq T(r, f)=S\left(r, f e^{g}\right)$, which is impossible, thus $a_{q} \equiv 0$. Repeating the above argument, one can obtain that $a_{i} \equiv 0$ for all $i=0,1, \ldots, m$. Hence the result follows. 
This completes the proof of Theorem 4 .

\section{Proof of Theorem 1 and 2}

In this section, we will prove Theorem 1 and 2 by using Theorem 4 .

Proof of Theorem 1 This follows immediately from part (4) of Theorem 4 and the fact that $T\left(r, \Gamma^{\prime} / \Gamma\right)=r+o(r)$ in [4].

Proof of Theorem 2. We consider the following two cases.

Case 1. If $\rho\left(e^{h}\right)<\infty$, then $\Gamma e^{h}$ is hypertranscendental over $\mathcal{M}_{0}$ (see p.271 of [3]). Actually, in this case, $h$ is a polynomial, hence it is not hard to see that $e^{h} \in A\left(\mathcal{M}_{0}\right)$ as $h^{\prime}=\left(e^{h}\right)^{\prime} / e^{h}$. If $\Gamma e^{h} \in A\left(\mathcal{M}_{0}\right)$, one can conclude that $\Gamma \in A\left(\mathcal{M}_{0}\right)$ which is a contradiction to the hypertranscendence of $\Gamma$ over $\mathcal{M}_{0}$.

Case 2. If $\rho\left(e^{h}\right)=\infty$, then $\Gamma \in \mathcal{S}_{\exp (h)}$, hence the result follows immediately from Theorem 4(5).

\section{ACKNOWLEDGEMENTS}

The first author was partially supported by a graduate studentship of HKU and the RGC grant 1731115. The second author was partially supported by the RGC grant 1731115 . The authors are very grateful to the referee for the valuable suggestions.

\section{REFERENCES}

[1] S. Bank. On the growth of meromorphic solutions of linear differential equations having arbitrary entire coefficients. Ann. Mat, Pura Appl., 107:279-289, 1976.

[2] S. Bank. Some results on Gamma function and other hypertranscendental functions. Proc. $R$. Soc. Edinb., 79A:335-341, 1977.

[3] S. Bank. Some Results on Hypertranscendental Meromorphic Functions. Monatsh. Math., 90:267-289, 1980.

[4] S. Bank and R. Kaufman. An extension of Hölder's theorem concerning the Gamma function. Funkcialaj Ekvacioj, 19:53-63, 1976.

[5] J. Clunie. The composition of entire and meromorphic functions. Mathematical Essays Dedicated to A. J. Macintyre. OH. Univ. Press, Athens, Ohio, 1970.

[6] F. Gross and C. F. Osgood. A simple proof of a theorem of Steinmetz. J. Math. Anal. Appl., 143:290-294, 1989.

[7] Y. Z. He and C. C. Yang. Meromorphic functions that do not satisfy algebraic differential equations. Acta Math. Sci. (Chinese), 11(3):343-348, 1991.

[8] D. Hilbert. Mathematische probleme. Arch.Math.Phys., 1:44-63, 213-317, 1901.

[9] O. Hölder. Über die Eigenschaft der $\Gamma$-Function, keiner algebraischen Differentialgleichung zu genügen. Math. Ann., 28:1-13, 1887.

[10] I. Laine. Nevanlinna theory and complex differential equations. Walter de Gruyter, Berlin, 1993.

[11] B. Q. Li. On reduction of functional-differential equations. Complex Variables, 31:311-324, 1996.

[12] B. Q. Li and Z. Ye. Algebraic differential equations with functional coefficients concerning $\zeta$ and Г. J. Differential Equations, 260:1456-1464, 2016. 
[13] L. Markus. Differential independence of $\Gamma$ and $\zeta$. J. Dynam. Differential Equations, 19:133$154,2007$.

[14] A. Z. Mokhon'ko. Estimates of Nevanlinna characteristics of algebroid functions and their applications to differential equations. Sib. Math. J., 23:80-88, 1982.

[15] N. Steinmetz. Über die Faktorisierbaren Losungen gewöhnlicher differential Gleichungen. Math. Z., 170:169-180, 1980.

Department of Mathematics, The University of Hong Kong, Pokfulam Road, HONG KONG

E-mail address: hjxmath@gmail.com

Department of Mathematics, The University of Hong Kong, Pokfulam Road, HONG KONG

E-mail address: ntw@maths.hku.hk 